\title{
Retrospective Study on Implantation of Autologous- Cultured Osteoblasts for the Treatment of Patients with Avascular Necrosis of the Femoral Head
}

This article was published in the following Dove Press journal: Orthopedic Research and Reviews

\author{
Gauresh Palekar' \\ HP Bhalodiya ${ }^{2}$ \\ Shreedhar Archik ${ }^{3}$ \\ Kalpesh Trivedi ${ }^{4}$ \\ 'Orthopedic Surgeon, Surya Orthopedic \\ Clinic, Mumbai, Maharashtra, India; \\ ${ }^{2}$ Orthopedic Surgeon, Walk-Fit Saviour \\ Hospital, Ahmedabad, Gujarat, India; \\ ${ }^{3}$ Orthopedic Surgeon, Dr. Sreedhar \\ Aarchik Orthopedic Care Clinic, Dadar \\ West, Mumbai, Maharashtra, India; \\ ${ }^{4}$ Arthroscopy Surgeon, Aastha \\ Arthroscopy and Joint Surgery Clinic, \\ Ahmedabad, Gujarat, India
}

Purpose: Osteonecrosis of the femoral head is a progressive and debilitating disease that causes pain, osteoarthritis and hip joint collapse, eventually necessitating hip replacement. This study evaluated the long-term outcomes of autologous adult live-cultured osteoblasts (AALCO) implantation in patients with osteonecrosis of femoral head.

Patients and Methods: In this retrospective multicenter study, we collated and analyzed data of patients $\geq 12$ years of age who underwent AALCO (OSSGROW ${ }^{\mathbb{R}}$ ) between 2010 and 2015 for the treatment of osteonecrosis of the femoral head.

Results: Data from 64 patients (101 hip joints) were assessed in this study. The mean \pm SD duration of disease since diagnosis of osteonecrosis was $7.4 \pm 1.6$ years. The mean follow-up duration was $6.3 \pm 1.4$ years. The mean VAS score ( $\mathrm{n}=98$ hips) reduced significantly from 58.8 \pm 13.8 to $32.2 \pm 32.1$ post-operatively (mean difference: $-26.5 \pm 35.2, \mathrm{p}=0.001$ ) and Harris hip score ( $\mathrm{n}=97 \mathrm{hips}$ ) also significantly improved from $47.1 \pm 12.3$ to $63.7 \pm 27.7$ post-operatively (mean difference:16.7 $\pm 28.7, \mathrm{p}=0.0001$ ). Following the AALCO treatment, 29 hips in 13 patients $(28.7 \%)$ underwent total hip replacement (THA), indicating that AALCO treatment could delay THA for $71.3 \%$ of hips. A total of $39.1 \%$ of hips diagnosed in early stage versus $60.8 \%$ in the late stage of osteonecrosis required THA. Overall, $60.4 \%$ of hips improved, $2 \%$ remained stable, and $37.6 \%$ progressed following the AALCO implantation. The condition of disease was found to have improved in $71.1 \%$ of patients in early stage (Grades I and II) versus 58\% in the late stage (Grades III and IV) of osteonecrosis.

Conclusion: Patients with osteonecrosis who received implantation of autologous-cultured osteoblasts using AALCO showed improvement in joint function and decrease in pain. Treatment with AALCO halted progression of osteonecrosis, preserved the natural hip, and eliminated the need for hip replacement surgeries in 7 out of 10 patients.

Keywords: autologous adult live-cultured osteoblasts, osteonecrosis, total hip replacement, femoral head

\section{Introduction}

Osteonecrosis of the femoral head is a progressive disorder that causes pain and often progresses to hip joint collapse, finally resulting in disabling arthritis. ${ }^{1,2}$ It occurs between 30 to 50 years of age, and prevails at a relatively younger age in Asians compared to their western counterparts. ${ }^{3}$ It is estimated that approximately 20,000-30,000 new cases of osteonecrosis are diagnosed in the United States each year, accounting for $10 \%$ of total hip arthroplasties performed. ${ }^{4}$ The Indian Society of Hip and Knee Surgeons has reported that more than 50\% of all hip replacements in India are performed for osteonecrosis. ${ }^{5}$ Many studies have reported
Correspondence: Gauresh Palekar Surya Orthopedic Clinic, I73/D, Sanzgiri Sadan, J S S Road, Mumbai, Maharashtra, 400004, India

Email drgauresh@hotmail.com
Orthopedic Research and Reviews 2021:13 15-23 
osteonecrosis to be more prevalent in men compared to women (3 or $5: 1){ }^{3}$ The underlying pathophysiology of osteonecrosis remains unclear; however, it is multifactorial and several traumatic and nontraumatic etiological factors may contribute to its development. Traumatic events that may cause osteonecrosis include femoral neck/head fracture, hip dislocation, or slipped capital femoral epiphysis. Nontraumatic factors include use of steroids, alcoholism, metabolic disorders such as Cushing's syndrome, and inherited disorders such as sickle cell disease. ${ }^{6,7}$ Besides the known traumatic and nontraumatic causes, some cases of osteonecrosis are idiopathic. ${ }^{1,8}$

Osteonecrosis of the femoral head may progress to secondary arthritis, and degeneration of articulating surface from advanced osteonecrosis necessitates total hip arthroplasty (THA). A primary treatment target of osteonecrosis of femoral head is to delay/prevent progression to osteoarthritis. Core decompression (CD) is the most widely used procedure in clinical practice; however, it has shown poor clinical outcomes, with up to $40 \%$ of patients having to undergo THA despite undergoing core decompression procedure. $^{8}$ Therefore, a more pathophysiological approach may be required to treat osteonecrosis of femoral head. Osteonecrosis is characterized by a reduction in the osteogenic progenitor cells, an increase in osteoblast death, and altered intramedullary vascular supply due to trauma. ${ }^{1}$ It was observed that the number and function of mesenchymal cells in hematopoietic tissue and stroma of the bone marrow decreased in osteonecrosis patients. ${ }^{2}$ This observation indicated potential for using bone marrow stromal cells for the treatment of osteonecrosis, and consequently, several clinical studies have demonstrated encouraging results. ${ }^{2}$ A meta-analysis also showed that treatment with cell therapy compared to core decompression alone increased Harris hip score, decreased necrotic area of femoral head and collapse of femoral head, and reduced THA conversion rate. ${ }^{9}$ However, a recent randomized study has shown that bone marrow cell implantation in addition to core decompression did not improve THA conversion rate in patients with grade 3 osteonecrosis. ${ }^{10}$ The ideal treatment goal for osteonecrosis is to facilitate new bone formation in the place of dead bone that can provide pain relief, cease disease progression, prevent joint collapse, and preserve the joint. The fact thatbone marrow aspirate consists of mesenchymal stem cells raised a possibility if bone marrow cells could be differentiated into bone forming cells or osteoblasts and characterized by bone alkaline phosphatase. In a randomized trial, autologous osteoblast implantation was shown to significantly delay the evolution to subchondral fracture and reduce pain compared to bone marrow aspirate. $^{11}$

OSSGROW $^{\circledR}$ (Regrow Biosciences Pvt Ltd., Mumbai, India) is a commercially available technology that involves implantation of autologous adult live-cultured osteoblasts (AALCO) derived from mesenchymal stem cells sourced from the bone marrow aspirate for osteonecrosis of the hip that received conditional marketing approval in India in March 2017. ${ }^{12}$ Here, we evaluated the efficacy of OSSGROW $^{\circledR}$ implantation technique by assessing retrospective data from patients with osteonecrosis who underwent the procedure. We also evaluated the correlation between Ficat-Arlet stages of osteonecrosis and clinical outcomes of the AALCO implantation procedure.

\section{Patients and Methods Study Design and Patient Selection}

This retrospective, observational, non-comparative study was conducted at 37 centers in India. We retrospectively reviewed the data of patients with osteonecrosis of the femoral head who had undergone OSSGROW ${ }^{\circledR}$ (AALCO) from 2010 to 2015. Key inclusion criteria were patients aged $\geq 12$ years with a confirmed diagnosis of osteonecrosis in one or both hip joints who had undergone AALCO implantation. Diagnosis, analysis, and classification of osteonecrosis were done according to Ficat-Arlet based on radiography, computed tomography (CT) scans, and magnetic resonance imaging (MRI) findings. Patients whose medical records were not complete or were lost to followup were excluded from the study.

The protocol was approved by the Institutional Ethics Committee - Regrow Biosciences Pvt Ltd. and as this study was a retrospective study, informed consent was not required to review medical records. We also sought permission from the head of the institutes/departments before data collection. Patient data confidentiality was maintained in this study.

\section{Study Procedure and Data Collection}

All the patients had undergone AALCO implantation on the recommendation of their consulting orthopedic surgeon after having received an explanation of the complications of osteonecrosis, the therapeutic options available, and the risks involved with the implantation procedure. Osteoblasts from patients were obtained from bone marrow aspiration from the posterior/superior iliac crest. 
Mesenchymal stem cells from the bone marrow were isolated and differentiated ex vivo into osteoblasts. Osteoblasts were then cultured for approximately 4 weeks under stringent laboratory conditions and multiplied up to 48 million osteoblasts (Figure 1). The cultured cells were implanted using a gel (Tisseel kit from Baxter) at the site of osteonecrosis through a minimally invasive surgery in a 3-step procedure: core decompression, curettage, and injection of osteoblasts (Figure 2).

Patients were operated on under spinal anesthesia. Core decompression tunnels were created into the subchondral necrotic lesion of the femoral head, approximately 2-3 mm away from the joint cartilage, by using 2.0-mm K-wires under fluoroscopic guidance through the greater trochanter and the femoral neck, and over drilled using trephine was performed by the centrally positioned K-wire. Cultured osteoblasts were injected following the curettage, and necrotic tissues were removed.
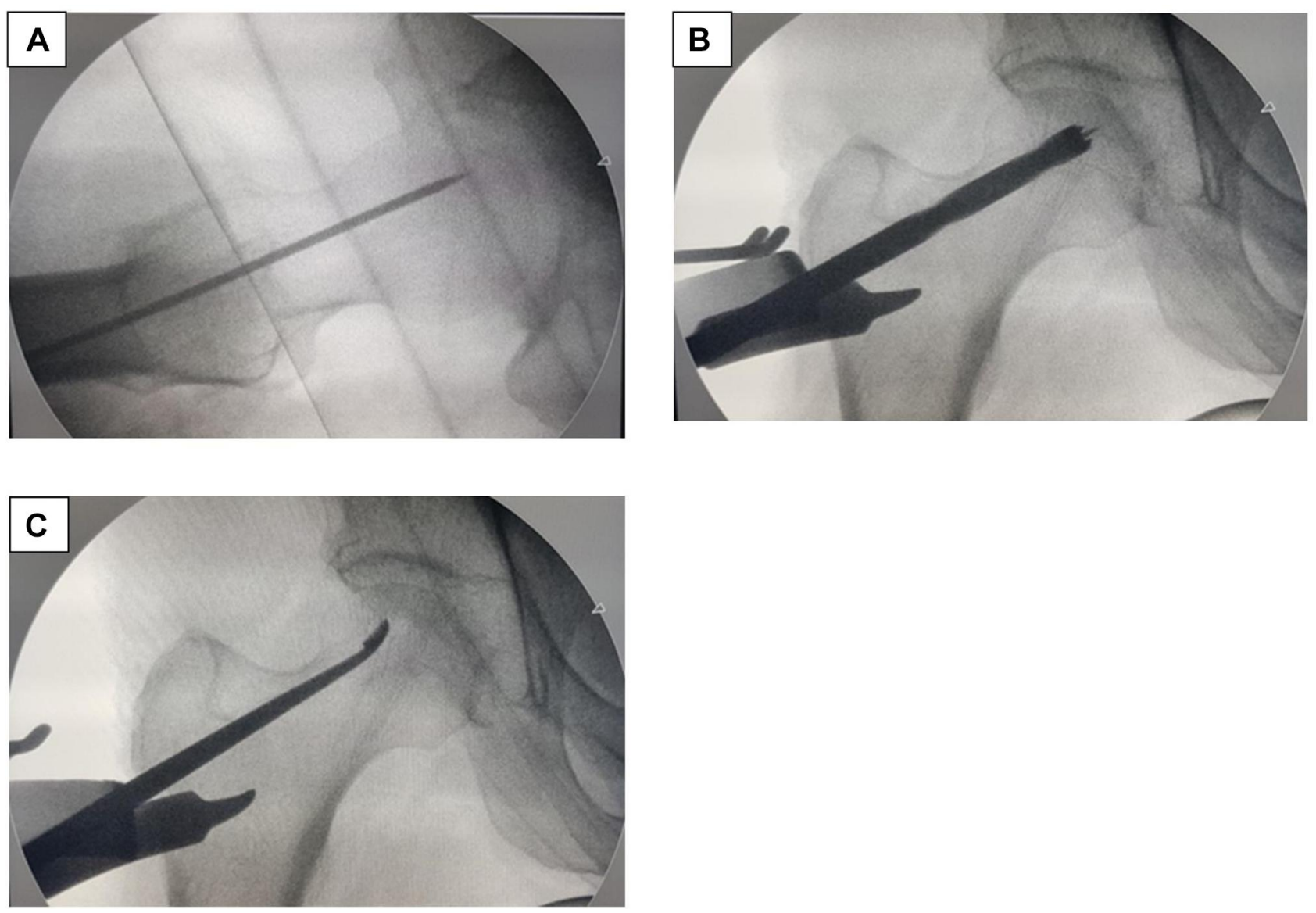

Figure I Microscopic image of osteoblast in culture used for the final cell product before cell implantation.

The patients had to undergo appropriate rehabilitation therapy after the implantation, which included complete bedrest for 4 weeks post-implantation. After 4 weeks, passive

Figure 2 Steps of osteoblasts implantation. (A) Step I - Insertion of guide wire in center of lesion as identified on the MRI. (B) Step 2 - Guide wire and 8mm cannulated drill for core decompression. The entry point of the guide wire is near the vastus ridge, to prevent a fracture due to stress-riser, greater width of femur and faster healing due to cancellous bone. (C) Step 3 - Curettage: a variety of angulated curettes is used to do forage (curettage to remove necrotic bone). This bone is sent for biopsy. 
lower limb exercises were performed for 2 weeks following post-implantation. Accordingly, non-weight bearing, partial weight bearing, and full weight bearing exercises were suggested as per the study protocol. Descriptive demographic and clinical data recorded before and after the procedure were collected from patient records. Past medical history, concomitant medications, and surgical treatments undertaken before and after the AALCO were recorded. Pre-existing risk factors for osteonecrosis such as steroid intake, alcohol consumption, comorbid conditions, or trauma were also noted.

\section{Study Outcomes}

Improvement in functional capacity and pain reduction were evaluated using Harris Hip Score (HHS) and visual analog scale (VAS) respectively at the time of pre- and postoperative consultations. Continued use of steroids or alcohol consumption after undergoing the AALCO implantation was recorded. The main outcome of the study was the need for THA (THA conversion rate). Based on these parameters, the treatment outcome was determined to be either improved (better score after AALCO implantation), stable (same condition as before AALCO implantation), or progressive (worse scores following AALCO implantation).

\section{Statistical Analysis}

Continuous and quantitative variables were summarized using descriptive statistics and compared using Student's $t$-test or nonparametric test, as applicable. Categorical data were presented as frequency count (n) and percentages (\%) and were compared using the $\chi^{2}$ test or Fisher's exact test. P-values $<0.05$ were considered significant. All analyses were performed using the SPSS version 10.0.

\section{Results}

Data from 64 patients were collected and analyzed as per the study protocol, and 101 hip joints were assessed. The age of patients ranged from 12-70 years and BMI ranged from $20.6-32 \mathrm{~kg} / \mathrm{m}^{2}$. The majority of the patients were men (79.7\%). The mean duration since diagnosis of osteonecrosis was $7.4 \pm 1.6$ years and the mean duration of AALCO treatment was $6.3 \pm 1.4$ years (Table 1). Unilateral involvement of the hip joint was seen in $42.2 \%$ of cases. Bilateral involvement of hip joints was seen in $57.8 \%$ of patients. The majority of hips diagnosed were grade III (42.1\%) and grade IV osteonecrosis (10.5\%). While the exact cause for osteonecrosis was not known (idiopathic) in $25 \%$ of patients, $35.9 \%$ of cases were linked to steroid use and $26.6 \%$ to alcohol abuse. Records of concomitant medications revealed
Table I Demographic Characteristics

\begin{tabular}{|c|c|}
\hline Parameters & $N=64$ \\
\hline Age (years) (mean $\pm \mathrm{SD})$ & $33.9 \pm 10.7$ \\
\hline \multicolumn{2}{|l|}{ Gender, N (\%) } \\
\hline Male & $51(79.7)$ \\
\hline $\mathrm{BMI}\left(\mathrm{kg} / \mathrm{m}^{2}\right)($ mean $\pm \mathrm{SD})$ & $25.2 \pm 2.3$ \\
\hline Disease duration (years) & $7.4 \pm 1.6$ \\
\hline Treatment duration (years) & $6.3 \pm 1.4$ \\
\hline No. of hips affected $(\mathrm{N})$ & 101 \\
\hline \multicolumn{2}{|l|}{ Location of osteonecrosis $(\mathrm{N}=\mathrm{I0I})$} \\
\hline Left hip & $50(52.6)$ \\
\hline Right hip & $45(47.4)$ \\
\hline \multicolumn{2}{|l|}{ Grade of osteonecrosis $(\mathrm{N}=95)$} \\
\hline I & $6(6.3)$ \\
\hline II & $39(4 I . I)$ \\
\hline III & $40(42.1)$ \\
\hline IV & $10(10.5)$ \\
\hline \multicolumn{2}{|l|}{ Causes of osteonecrosis $(N=64)$} \\
\hline Alcohol & $17(26.6)$ \\
\hline Smoking & $7(10.9)$ \\
\hline Idiopathic & $16(25)$ \\
\hline \multicolumn{2}{|l|}{ Steroid use for pre-existing illness } \\
\hline Unknown & $23(35.9)$ \\
\hline Leukemia & I (I.6) \\
\hline Systemic lupus erythematosus & I (I.6) \\
\hline Rheumatoid arthritis & I ( 1.6$)$ \\
\hline Tuberculosis & $\mathrm{I}(\mathrm{l} .6)$ \\
\hline Trauma & $9(14.1)$ \\
\hline \multicolumn{2}{|l|}{ Concomitant medications, $n(\%)(\mathrm{N}=37)$} \\
\hline Analgesics & 34 (91.9) \\
\hline Ayurvedic medication & $3(8.1)$ \\
\hline
\end{tabular}

Abbreviations: BMI, body mass index; SD, standard deviation.

that $91.9 \%$ of patients were on analgesics, $8.1 \%$ were on ayurvedic treatment, and 1 patient took bisphosphonate.

\section{Changes in VAS Score and HHS Score}

A total of 98 hip joints were assessed as data of 3 patients were not available for changes in mean VAS scores (improvement in pain), before and after the AALCO implantation. As shown in Figure 3A, the mean VAS score reduced significantly after a mean 6.3 years of AALCO treatment compared to the baseline (32.2 \pm 32.1 vs $58.8 \pm 13.8$; mean difference: $-26.5 \pm$ $35.2, \mathrm{p}=0.001)$ indicating significant improvement in pain. Similarly, HHS also improved post-operatively $(47.1 \pm 12.3 \mathrm{vs}$ $63.7 \pm 27.7$; mean difference: $16.7 \pm 28.7, \mathrm{p}=0.001$ ) showing functional improvement of patients. We categorized patients 
A

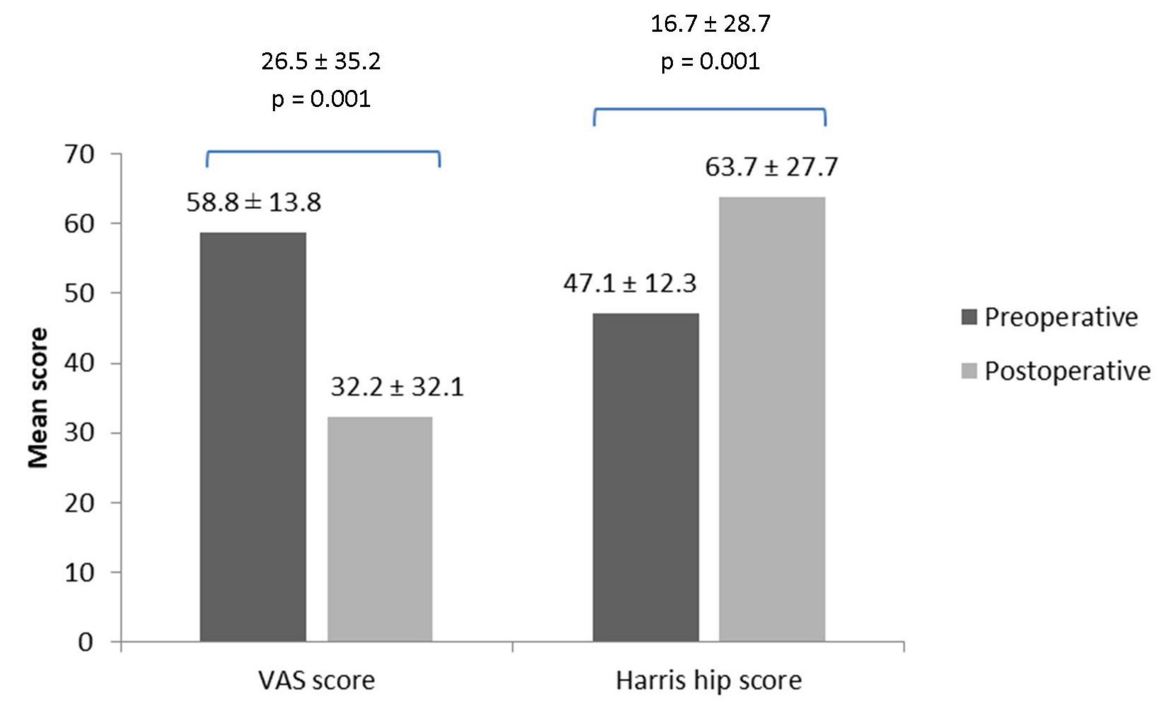

B

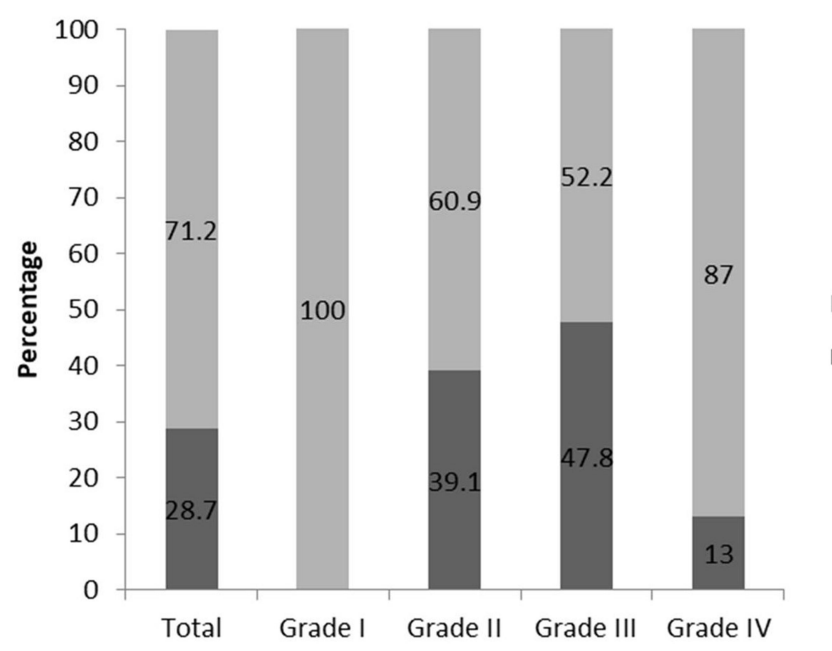

Did not need surgery

Need hip replacement surgery

Figure 3 (A) Changes in visual analog scale (VAS) and Harris hip scores. (B) Need for hip replacement surgery in different grades of osteonecrosis. (Osteonecrosis graded according to Association Research Circulation Osseous criteria).

based on their HSS score (<70: poor, 70-80: fair, 80-90: good, 90-100: excellent). At baseline, 96 hips (98\%) had HSS score of $<70$, each of the two remaining hips had scores of $<80$ and $<90$, respectively. Improvement in HSS scores was seen at follow-up with 42 hips (43.3\%) with HSS <70, 11 (11.3\%) with 70-80, $26(26.8 \%)$ with 80-90, and $18(18.6 \%)$ with HSS scores of $90-100$.

\section{Need for Surgery}

The mean follow-up period since diagnosis of osteonecrosis was 6.3 years (range 4-9 years). Following AALCO treatment, $29(28.7 \%)$ hips underwent THA, indicating that AALCO treatment could prevent and delay THA for $71.3 \%$ of hips. The mean time to THA was $3.2 \pm 2.0$ years (range: 1-9 years). A total of $9(39.1 \%)$ grade II, 11 $(47.8 \%)$ grade III, and $3(13 \%)$ grade IV hip joints required THA surgery (Figure 3B). In other words, AALCO treatment could delay THA for up to 3 years in $80 \%$ of hips in early stage osteonecrosis (Grades I and II) and $72 \%$ of hips in late stage osteonecrosis (Grades III and IV). Univariate analysis showed that the age of the patient, BMI, gender of patients, the side of osteonecrosis, and 


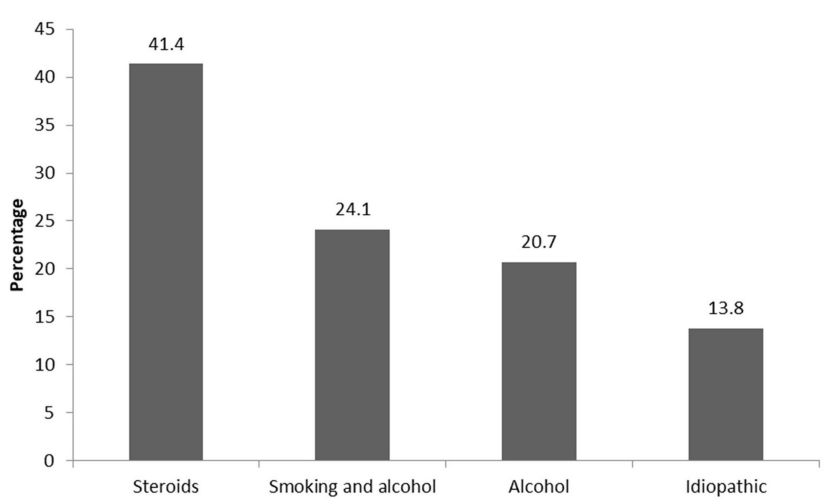

A

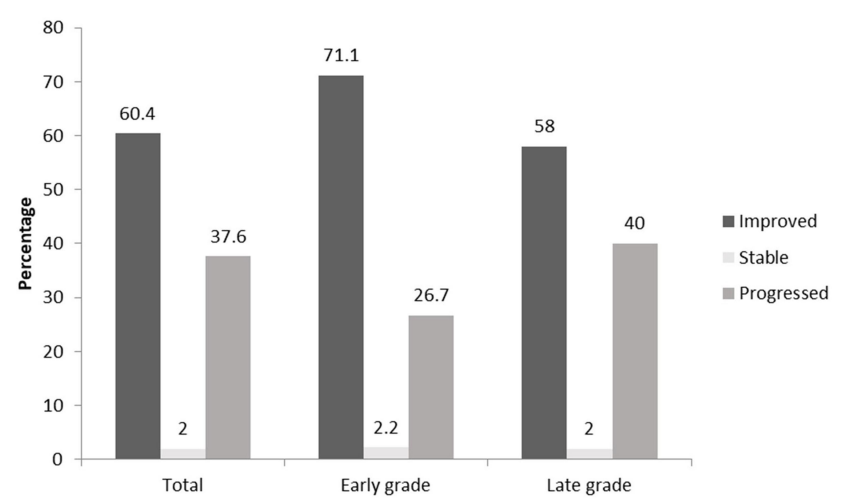

B

Figure 4 (A) Need for hip replacement stratified as per etiology of osteonecrosis. (B) Overall outcome stratified as per the grades of osteonecrosis. Abbreviations: RA, rheumatoid arthritis; SLE, systemic lupus erythematous.

duration of disease had no effect on the clinical success of the procedure. Following AALCO treatment, 35.9\% of patients continued using steroids and $29.7 \%$ continued with alcohol consumption. Of the total 29 hip joints that required surgery at follow-up, $20.7 \%$ and $41.4 \%$ had an associated etiology of alcohol consumption and steroid intake, respectively (Figure 4A). Overall, a significantly greater number of patients with underlying etiologies of alcohol consumption, smoking, or taking steroids required THA compared to those without these etiologies (14 [37.8\%] vs 3 [11.1\%], $\mathrm{p}=0.017)$.

\section{Outcome of AALCO Procedure}

Based on the pre- and post-operative data, the condition of $65.6 \%$ of patients improved and $1.6 \%$ remained stable following AALCO treatment. Overall, the condition of $65.9 \%$ of hips $(56 / 85)$ in grade I to grade III improved (Figure 4B). For quick reference, the pre- and postoperative radiograph images for a given patient are presented in Figure 5.

\section{Discussion}

We retrospectively studied the clinical outcomes of AALCO treatment. Our results showed that there was a reduction in pain and improvement in joint function following AALCO implantation, as was evident from a statistically significant reduction in the mean VAS score and increase in the HHS score. Of all the hips that underwent the AALCO implantation, 60\% improved and $38 \%$ worsened with a THA conversion rate of $28 \%$.

AALCO is a minimally invasive, surgical 3-step procedure with each step contributing significantly to the overall effectiveness of the treatment. The first step is core decompression that reduces pressure allowing increased blood flow. In the second step, the necrotic bone is debrided by a curette that promotes new bone formation. The third and most important step is implantation of osteoblasts that form new bone. The THA conversion rate is reported lower with core decompression compared to natural progression of disease, but approximately $40 \%$ of patients still required THA. ${ }^{8}$ Bone marrow cell therapy was shown to improve the THA conversion rate further. ${ }^{9}$ In a recent randomized trial, implantation of autologous bone marrow aspirate concentrate did not show any improvement in patients with grade 3 osteonecrosis. ${ }^{10}$ In our study, AALCO implantation avoided THA in $72 \%$ of hips in late grade osteonecrosis, suggesting that the technique may even benefit patients in advanced stages of disease; however, our results are limited by the relatively small numbers of patients belonging to each stage.

The differences in the THA conversion may not be directly comparable to those with others may be due to the diversity in the presentation of patients, differences in the follow-up period, or the AALCO technique. ${ }^{13,14}$ The THA conversion rate certainly remains low with AALCO treatment compared to $75 \%$ THA conversion rate reported in patients with natural progression to osteoarthritis resulting from osteonecrosis of the femoral head. ${ }^{15,16}$ A randomized study found autologous osteoblastic cells implantation to be more efficacious than bone marrow implantation as an adjunct to core decompression. The disease progression rate was found to be $20 \%$ in patients who had undergone autologous osteoblasts implantation vs $47 \%$ in patients in the bone marrow implantation group. ${ }^{11}$ Bone alkaline phosphatase-characterized osteoblasts have better regenerative potential compared to heterogeneous bone marrow cells. ${ }^{17,18}$ Use 

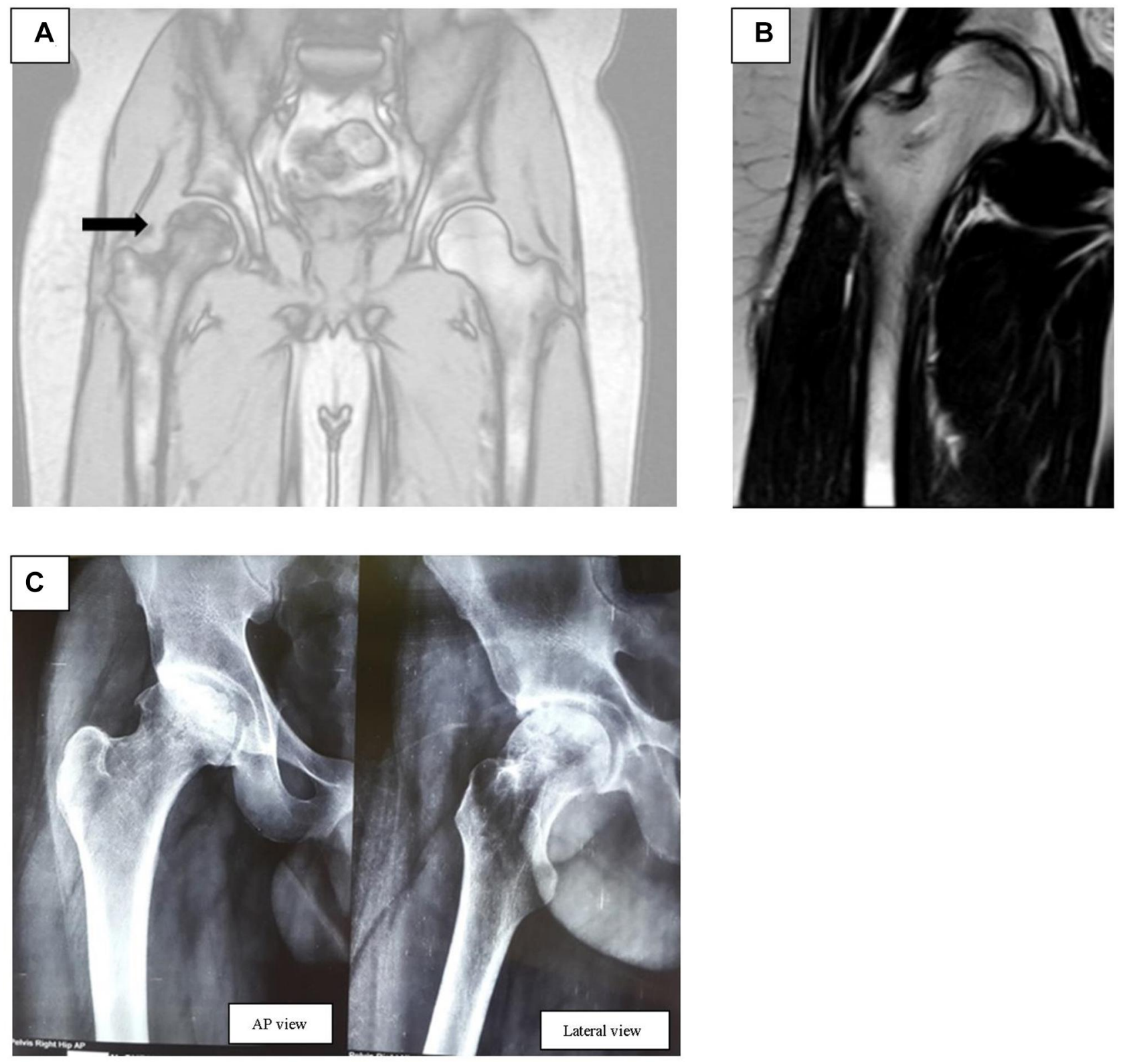

Figure 5 Pre- and posttransplantation MRI and X-ray images (A): pre-operative MRI - (male patient [35 years]): Ficat and Arlet Stage II B with a subchondral fracture of right hip with a large anterolateral lesion (arrow) involving more than $40 \%$ of femoral head and less than $2 \mathrm{~mm}$ depression at high risk of collapse. Etiology is post steroid AVN. (B) Post-operative MRI at 5 months post-surgery. (C) Post-operative X-ray at 4 years after surgery; anteroposterior (AP) view and lateral view.

of these characterized cells could explain the favorable outcomes of AALCO implantation in our study.

Intake of alcohol and/or steroids is known to adversely affect bone renewal by causing an imbalance between the normal progenitor cells and the fat-storing bone marrow progenitor cells. ${ }^{1,19,20}$ The latter phenotype also leads to fat embolism and arteriosclerosis reducing the blood supply to necrotic tissues. ${ }^{1,19,20}$ In our study, alcohol and steroid intake were associated with occurrence of osteonecrosis of the femoral head in more than a quarter of patients. These results highlight the adverse impact of alcohol and steroid intake on the progression of osteonecrosis that is already evident in the literature in the pathogenesis of osteonecrosis. ${ }^{21-25}$ As expected, THA conversion rate was also higher among patients who consumed alcohol and/or used steroids compared to those who did not in our study, signifying the adverse impact of alcohol and steroids on the AALCO treatment outcomes. However, a consensus on the specific mechanisms leading to these observations is yet to be reached.

A major limitation of our study was the retrospective data collection, and the lack of assessments of radiographic progression of the affected hips. 


\section{Conclusion}

The results of this study substantiate the therapeutic potential for AALCO in improving clinical outcomes in terms of pain and functional activity, and reducing the risk of disease progression and the need for THA in patients with osteonecrosis. However, this study was limited by the small sample size and the retrospective data collection limiting the power of study for some subgroup comparisons. Further, clinical studies and long-term trials are warranted to confirm the findings of this study.

\section{Acknowledgments}

Authors acknowledge CBCC Global Research for providing medical writing and submission support funded by Regrow Biosciences Pvt. Ltd.

\section{Disclosure}

The authors report no conflicts of interest in this work.

\section{References}

1. Hernigou P, Poignard A, Zilber S, Rouard H. Cell therapy of hip osteonecrosis with autologous bone marrow grafting. Indian J Orthop. 2009;43(1):40-45. doi:10.4103/0019-5413.45322

2. Gangji V, Hauzeur J-P, Matos C, De Maertelaer V, Toungouz M, Lambermont M. Treatment of osteonecrosis of the femoral head with implantation of autologous bone-marrow cells: a pilot study. $J$ Bone Joint Surg Am. 2004;86(6):1153-1160. doi:10.2106/00004623-2004 06000-00006

3. Vardhan H, Tripathy SK, Sen RK, Aggarwal S, Goyal T. Epidemiological profile of femoral head osteonecrosis in the North Indian population. Indian $J$ Orthop. 2018;52(2):140-146. doi:10.4103/ortho.IJOrtho_292_16

4. Moya-Angeler J, Gianakos AL, Villa JC, Ni A, Lane JM. Current concepts on osteonecrosis of the femoral head. World J Orthop. 2015;6(8):590-601. doi:10.5312/wjo.v6.i8.590

5. ISHKS registry. Available from: http://www.ishks.com/pdf/ISHKSregistry-2019.pdf. Accessed December 17, 2020. 2019.

6. Xie XH, Wang XL, Yang HL, Zhao DW, Qin L. Steroid-associated osteonecrosis: epidemiology, pathophysiology, animal model, prevention, and potential treatments (an overview). J Orthop Translat. 2015;3(2):58-70. doi:10.1016/j.jot.2014.12.002

7. Jaffré C, Rochefort GY. Alcohol-induced Osteonecrosis-dose and duration effects. Int J Exp Pathol. 2012;93(1):78-79. doi:10.1111/ j.1365-2613.2011.00798_1.x

8. Houdek MT, Wyles CC, Martin JR, Sierra RJ. Stem cell treatment for avascular necrosis of the femoral head: current perspectives. Stem Cells Cloning. 2014;7:65-70. doi:10.2147/SCCAA.S36584

9. Xu S, Zhang L, Jin H, et al. Autologous stem cells combined core decompression for treatment of avascular necrosis of the femoral head: a systematic meta-analysis. Biomed Res Int. 2017;20 17:6136205. doi:10.1155/2017/6136205

10. Hauzeur JP, De Maertelaer V, Baudoux E, Malaise M, Beguin Y, Gangji V. Inefficacy of autologous bone marrow concentrate in stage three osteonecrosis: a randomized controlled double-blind trial. Int Orthop. 2018;42(7):1429-1435. doi:10.1007/s00264-017-3650-8
11. Hauzeur JP, Toungouz M, Lechanteur C, et al. Autologous osteoblastic cells (PREOBy) versus concentrated bone marrow implantation in osteonecrosis of the femoral head: a randomized study. Revue de Chirurgie Orthopédique et Traumatologique. 2016;102(7):S73. doi:10.1016/j.rcot.2016.08.002

12. Cuende N, Rasko JEJ, Koh MB, Dominici M, Ikonomou L. Cell, tissue and gene products with marketing authorization in 2018 worldwide. Cytotherapy. 2018;20(11):1401-1413. doi:10.1016/j. jcyt.2018.09.010

13. Pepke W, Kasten P, Beckmann NA, Janicki P, Egermann M. Core decompression and autologous bone marrow concentrate for treatment of femoral head osteonecrosis: a randomized prospective study. Orthop Rev (Pavia). 2016;8(1):6162. doi:10.4081/or.2016.6162

14. Zhao D, Cui D, Wang B, et al. Treatment of early stage osteonecrosis of the femoral head with autologous implantation of bone marrow-derived and cultured mesenchymal stem cells. Bone. 2012;50(1):325-330. doi:10.1016/j.bone.2011.11.002

15. Hernigou P, Habibi A, Bachir D, Galacteros F. The natural history of asymptomatic osteonecrosis of the femoral head in adults with sickle cell disease. J Bone Joint Surg Am. 2006;88(12):2565-2572. doi:10.2106/00004623-200612000-00002

16. Tomaru Y, Yoshioka T, Sugaya H, et al. Ten-year results of concentrated autologous bone marrow aspirate transplantation for osteonecrosis of the femoral head: a retrospective study. BMC Musculoskelet Disord. 2019;20(1):410. doi:10.1186/s12891-019-2797-4

17. Birmingham E, Niebur G, McHugh PE. Osteogenic differentiation of mesenchymal stem cells is regulated by osteocyte and osteoblast cells in a simplified bone niche. Eur Cell Mater. 2012;23:13-27. doi:10.22203/eCM.v023a02

18. Prins H-J, Braat AK, Gawlitta D, et al. In vitro induction of alkaline phosphatase levels predicts in vivo bone forming capacity of human bone marrow stromal cells. Stem Cell Res. 2014;12(2):428-440. doi:10.1016/j.scr.2013.12.001

19. Cui Q, Wang GJ, Balian G. Steroid-induced adipogenesis in a pluripotential cell line from bone marrow. J Bone Joint Surg Am. 1997;79(7):1054-1063. doi:10.2106/00004623-199707000-00012

20. Hernigou P, Beaujean F, Lambotte J. Decrease in the mesenchymal stem-cell pool in the proximal femur in corticosteroid-induced osteonecrosis. $J$ Bone Joint Surg Br. 1999;81(2):349-355. doi:10.1302/0301-620X.81B2.0810349

21. Sakaguchi M, Tanaka T, Fukushima W, Kubo T, Hirota Y. Impact of oral corticosteroid use for idiopathic osteonecrosis of the femoral head: a nationwide multicenter case-control study in Japan. J Orthop Sci. 2010;15(2):185-191. doi:10.1007/s00776-009-1439-3

22. Kubo T, Ueshima K, Saito M, Ishida M, Arai Y, Fujiwara H. Clinical and basic research on steroid-induced osteonecrosis of the femoral head in Japan. J Orthop Sci. 2016;21(4):407-413. doi:10.1016/j. jos.2016.03.008

23. Cooper C, Steinbuch M, Stevenson R, Miday R, Watts N. The epidemiology of osteonecrosis: findings from the GPRD and THIN databases in the UK. Osteoporos Int. 2010;21(4):569-577. doi:10.1007/s00198-009-1003-1

24. Fukushima W, Fujioka M, Kubo T, Tamakoshi A, Nagai M, Hirota Y. Nationwide epidemiologic survey of idiopathic osteonecrosis of the femoral head. Clin Orthop Relat Res. 2010;468(10):2715-2724. doi:10.1007/s11999-010-1292-X

25. Kang JS, Park S, Song JH, Jung YY, Cho MR, Rhyu KH. Prevalence of osteonecrosis of the femoral head: a nationwide epidemiologic analysis in Korea. $J$ Arthroplasty. 2009;24(8):1178-1183. doi:10.1016/j.arth.2009.05.022 


\section{Publish your work in this journal}

Orthopedic Research and Reviews is an international, peer-reviewed, open access journal that focusing on the patho-physiology of the musculoskeletal system, trauma, surgery and other corrective interventions to restore mobility and function. Advances in new technologies, materials, techniques and pharmacological agents are particularly welcome. The manuscript management system is completely online and includes a very quick and fair peer-review system, which is all easy to use. Visit http://www.dovepress.com/testimonials.php to read real quotes from published authors. 